\title{
ERK Family Gene Mutation
}

National Cancer Institute

\section{Source}

National Cancer Institute. ERK Family Gene Mutation. NCI Thesaurus. Code C136423.

A change in the nucleotide sequence in a ERK family gene. 